\title{
Image-fusion Technique in Microvascular Decompression Surgery with 3D Constructive Interference in Steady-state and Modified 3D Time of Flight MR Images: Superior Detection of Possible Offending Vessels over Conventional Imaging
}

\author{
Shin Heon Lee, Taek Kyun Nam, Ki-Su Park, Yong-Sook Park, Seung Won Park, \\ Jeong-Taik Kwon, Young-Baeg Kim \\ Department of Neurosurgery, Chung-Ang University Hospital, Chung-Ang University College of Medicine, Seoul, Korea
}

\begin{abstract}
Objective: To assess the value of fusion imaging with three-dimensional (3D) constructive interference in steady-state (CISS) sequence magnetic resonance (MR) imaging and modified 3D time of flight (TOF) MR angiography (MRA) in the visualization of possible offending vessels in patients with neurovascular compression.

Methods: Pre-operative MR images of 15 consecutive patients who underwent surgical microvascular decompression for unilateral trigeminal neuralgia or hemifacial spasm were retrospectively collected. All patients were examined with 3D CISS sequence magnetic resonance (MR) imaging and 3D TOF MRA, and images were imported to OsiriX MD imaging software. The 3D TOF MR images were modified to exaggerate the arteries and fused with the 3D CISS MR images. We matched the images with intra-operative findings and post-operatively compared the offender prediction value between conventional MR images and fused MR images.

Results: Fused images showed spatial relationships between arterial vessels and nerves more clearly than conventional images. Of the 15 patients in the study, 11 cases $(73.3 \%)$ were found identical to the prediction by using fused MR images. The fused images revealed a secondary possible candidate offender, and 5 cases were predicted to have another possible offender. Furthermore, 3 cases could be predicted by fused MR images, whereas not detected by conventional images. Conclusion: The image-fusion technique can provide pre-operative information of possible offenders, which can be used to detect and localize an additional offending vessel during planning for cases with hidden vascular contacts on conventional MR images.
\end{abstract}

Key Words: Magnetic resonance imaging $\cdot$ Image processing $\cdot$ Computer-assisted $\cdot$ Microvascular decompression surgery $\cdot$ Trigeminal neuralgia $\cdot$ Hemifacial spasm

\section{INTRODUCTION}

Pre-operative anatomic evaluation with magnetic resonance (MR) imaging is widely done to precisely assess neurovascular relationships. Optimal images for evaluation of neurovascular relationships include high-resolution three-dimensional (3D) MR angiography (MRA) sequences, such as 3D time of flight (TOF) MRA, which allow visualization of small vessels, and high-resolution heavily T2 weighted image (T2-WI) 3D sequences with a very high cerebralspinal fluid (CSF) tissue contrast, such as 3D constructive interference in steady-state (CISS)

Corresponding author: Taek Kyun Nam

Department of Neurosurgery, Chung-Ang University Hospital, Chung-Ang

University College of Medicine, 102, Heukseok-ro, Dongjak-gu, Seoul

06973, Korea

Tel: +82-2-6299-3188, Fax: +82-2-6299-2064

E-mail:nsnam@cau.ac.kr sequence MR imaging ${ }^{1-3,-9,11,12)}$. Fused images derived from these two techniques can yield numerous benefits. Both the vessels and the cranial nerve are visualized with superior clarity using the image-fusion technique, and locating contact between the vessel wall and nerve can be more accurately pinpointed.

The purpose of this study is to compare the diagnostic value of the image-fusion technique with conventional MR imaging in patients with trigeminal neuralgia (TN) or hemifacial spasm (HFS) and investigate the efficacy of the image-fusion technique.

\section{MATERIALS AND METHODS}

\section{Patients}

Fifteen consecutive patients who underwent microvascular 
decompression (MVD) surgery between July 2011 and October 2015 for unilateral TN or HFS at the Chung-Ang University Hospital were included in the study. Clinical records and pre-operative MR images were retrospectively collected and reviewed for all patients.

\section{MR Imaging}

All patients included in the study were examined with a 1.5-T scanner (Magnetom Avanto; Siemens Healthcare, Erlangen, Germany), which used a body coil with contrast medium. Both 3D CISS and 3D TOF images were scanned by axial section. The centers of the slabs were in the same position for both sequences.

\section{Image Fusion}

Image fusion was performed on an Apple Macintosh iMac running OS X and OsiriX MD (Version 7.0, Pixmeo, Bernex, Switzerland) imaging software. The 3D TOF images were modified to exaggerate the arteries. Using OsiriX MD imaging software, the Color Look Up Table (CLUT) was changed to VR red vessels. Secondarily, window level (WL) value was increased from 290 to 430 and window width (WW) value is decreased from 470 to 200 . Finally, the modified 3D TOF

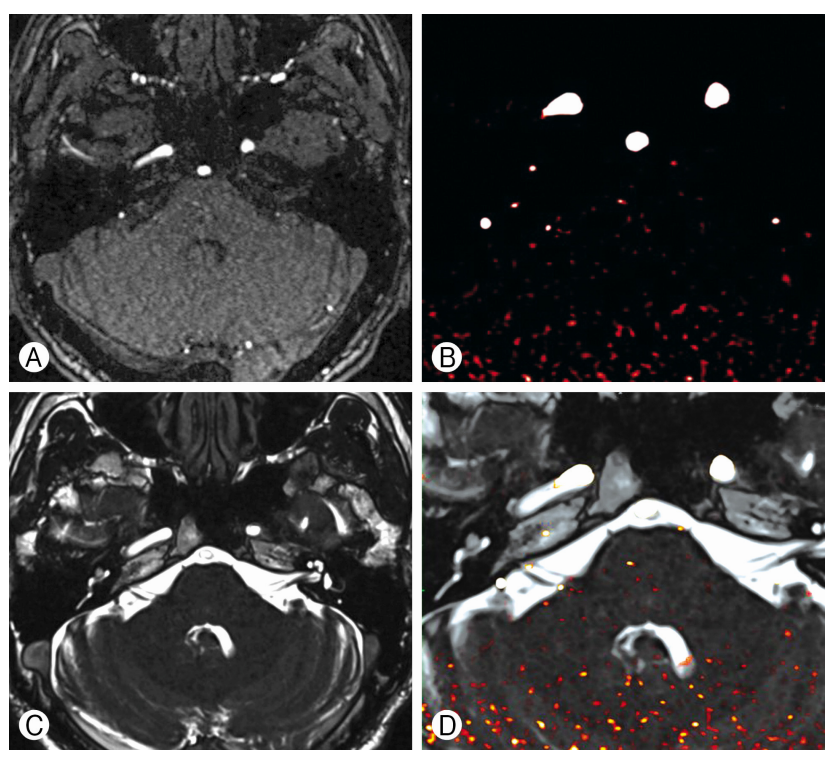

Fig. 1. Process of the image-fusion technique. (A) Three-dimensional (3D) time of flight (TOF) magnetic resonance (MR) image of the patient, (B) 3D TOF MR image with augmented vessel lumen modified by OsiriX imaging software, (C) 3D constructive interference in steady-state (CISS) MR image of the patient, (D) Augmented 3D TOF MR image fused with 3D CISS MR image demonstrates two separate vessels between facial and vestibular nerve. images were fused with the 3D CISS images (Fig. 1).

\section{Surgical Treatment}

All patients included in the study underwent MVD surgery. For each patient, MVD via a retro-sigmoid approach with exploration of the root entry zone (REZ) of the affected nerve was performed.

\section{Evaluation of Neurovascular Compression}

Post-operatively, the fused images and conventional MR images were separately compared with the video-documented intra-operative microscopic findings, with special attention paid to the identity and nature of the offending vessel. To avoid prejudice, the images were matched and then reviewed by two different neurosurgeons who were blind to patients' symptoms. During a two-weeks interval, both images were cross-checked, and concordant results were compared.

\section{RESULTS}

\section{Demographic Data}

Of the 15 patients included in the study, 10 were men (66.7\%) and 5 were women (33.3\%). The mean patient age was 53 years, with ages ranging from 33 to 67 . Seven patients (46.7\%) reported symptoms on the right side and 8 patients $(53.3 \%)$ reported symptoms on the left side (Table 1).

\section{Conventional MR Imaging Data}

Conventional MR images were reviewed. For the 15 patients in the study, the causative vessels identified were the posterior inferior cerebellar artery (PICA; 5 patients), the anterior inferior cerebellar artery (AICA; 2 patients), the vein (2 patients) and the vertebral artery (VA; 1 patient). In 1 patient, both the AICA and the PICA were predicted as the offender, and in 1 patient both the AICA and the VA were predicted as the offender. In 2 patients, the offenders were detected but the origins of the vessels were undefined, and in 1 patient no causative vessel contact was found in the MR image (Table 2).

\section{Image Fusion Data}

The MR images for all 15 patients were successfully fused using OsiriX MD imaging software. After image processing, the spatial relationships between the vessels and nerves were 
Table 1. Predictive value of conventional and fused images

\begin{tabular}{|c|c|c|c|c|c|c|c|c|c|}
\hline $\mathrm{TN} / \mathrm{HFS}$ & $R / L$ & Sex & Age & Conventional & Fusion & IntraOP & $\begin{array}{l}\text { Predictive value } \\
\text { (CI) }\end{array}$ & $\begin{array}{l}\text { Predictive value } \\
\text { (FI) }\end{array}$ & $\begin{array}{c}\text { Concordance } \\
\text { (Cl-FI) }\end{array}$ \\
\hline \multirow[t]{4}{*}{ TN } & $R$ & $M$ & 48 & Vein & Not definite & SCA & $x$ & $x$ & \\
\hline & & $\mathrm{F}$ & 54 & Not definite & Small REZ a. & Small REZ a. & $x$ & $\mathrm{O}^{*}$ & \\
\hline & & $M$ & 62 & Vein & Not definite & Vein & O & $x$ & \\
\hline & $\mathrm{L}$ & $\mathrm{F}$ & 63 & AICA, PICA & PICA & PICA & O & O & O \\
\hline \multirow[t]{11}{*}{ HFS } & R & $M$ & 58 & $\mathrm{PICA}$ & PICA, VA & PICA & $\mathrm{O}$ & $\mathrm{O}$ & O \\
\hline & & $M$ & 54 & AlCA & $\mathrm{AlCA}$ & AICA & O & O & O \\
\hline & & $\mathrm{F}$ & 50 & $\mathrm{PICA}$ & Not definite & PICA & O & $x$ & \\
\hline & & $M$ & 42 & $\mathrm{PICA}$ & PICA, Small REZ a. & Small REZ a. & $x$ & $\mathrm{O}^{*}$ & \\
\hline & $\mathrm{L}$ & $M$ & 59 & $\mathrm{PICA}$ & PICA & $\mathrm{PICA}$ & O & O & O \\
\hline & & $\mathrm{F}$ & 59 & $\mathrm{PICA}$ & PICA, VA & VA & $x$ & $\mathrm{O}^{*}$ & \\
\hline & & $M$ & 33 & No contact & PICA & $\mathrm{AlCA}$ & $x$ & $x$ & \\
\hline & & $M$ & 61 & VA & VA & VA & O & O & O \\
\hline & & $M$ & 46 & AlCA, VA & AlCA, VA & AlCA & 0 & 0 & 0 \\
\hline & & $M$ & 34 & Not definite & $\mathrm{PICA}$ & PICA & $x$ & $\mathrm{O}^{*}$ & \\
\hline & & $\mathrm{F}$ & 67 & $\mathrm{AlCA}$ & AICA, PICA & $\mathrm{AlCA}$ & O & O & O \\
\hline Total & 15 & & & & & & $9 / 15$ & $11 / 15$ & $7 / 15$ \\
\hline
\end{tabular}

"Cases exclusively predicted by fused images.

HFS: hemifacial spasm; TN: trigeminal neuralgia; R: right; L: left; $M$ : male; F: female; AICA: anterior inferior cerebellar artery; PICA: posterior inferior cerebellar artery; VA: vertebral artery; REZ: root entry zone; IntraOP: intra-operative; Cl: conventional image; $\mathrm{Fl}$ : fused image.

evaluated in each case. Of the 15 patients in the study, the causative vessels were identified as the PICA in 4 patients, the AICA in 1 patient, the VA in 1 patient and the small REZ artery in 1 patient. In 2 patients, both the PICA and the VA were predicted as offenders; in 1 patient, both the AICA and the PICA were predicted as offenders; in 1 patient, both the AICA and the VA were predicted as offenders; and in 1 patient, both the PICA and the small REZ artery were predicted as offenders. In 3 patients, the offenders were detected but the origins of the vessels were undefined (Table 2).

\section{Surgical Data}

Of the 15 patients predicted to have neurovascular compression, all patients were found to have compression by an arterial or venous structure in the operative view. The causative vessels for neurovascular compression were intra-operatively found and identified as the PICA in 5 patients, the AICA in 4 patients, the VA in 2 patients, the small REZ artery in 2 patients, the superior cerebellar artery (SCA) in 1 patient and the vein in 1 patient (Table 2).

\section{Predictive Value}

The predictive values between the two images were different.
Table 2. Predicted offending vessels in magnetic resonance images and intra-operative findings

\begin{tabular}{lccc}
\hline \hline & Conventional & Fusion & IntraOP \\
\hline PICA & 5 & 4 & 5 \\
AICA & 2 & 1 & 4 \\
SCA & 0 & 0 & 1 \\
VA & 1 & 1 & 2 \\
Vein & 2 & 0 & 1 \\
REZ a. & 0 & 1 & 2 \\
AICA + PICA & $1^{*}$ & $1^{*}$ & 0 \\
AICA + VA & $1^{*}$ & $1^{*}$ & 0 \\
PICA + VA & 0 & $2^{*}$ & 0 \\
PICA + REZ a. & 0 & $1^{*}$ & 0 \\
Not definite & 2 & 3 & 0 \\
No vessel & 1 & 0 & 0 \\
${ }^{*} 2^{\text {nd }}$ offender prediction & 2 & 5 & \\
\hline PICA: posterior inferior cerber & arter &
\end{tabular}

PICA: posterior inferior cerebellar artery; AICA: anterior inferior cerebellar artery; SCA: superior cerebellar artery; VA: vertebral artery; REZ: root entry zone; IntraOP: intra-operative.

Of the 15 patients predicted to have neurovascular compression, 9 cases (60.0\%) were predicted by conventional images. In contrast, 11 cases $(73.3 \%)$ were found identical with the prediction using fused MR images. And, among them, 7 cases 

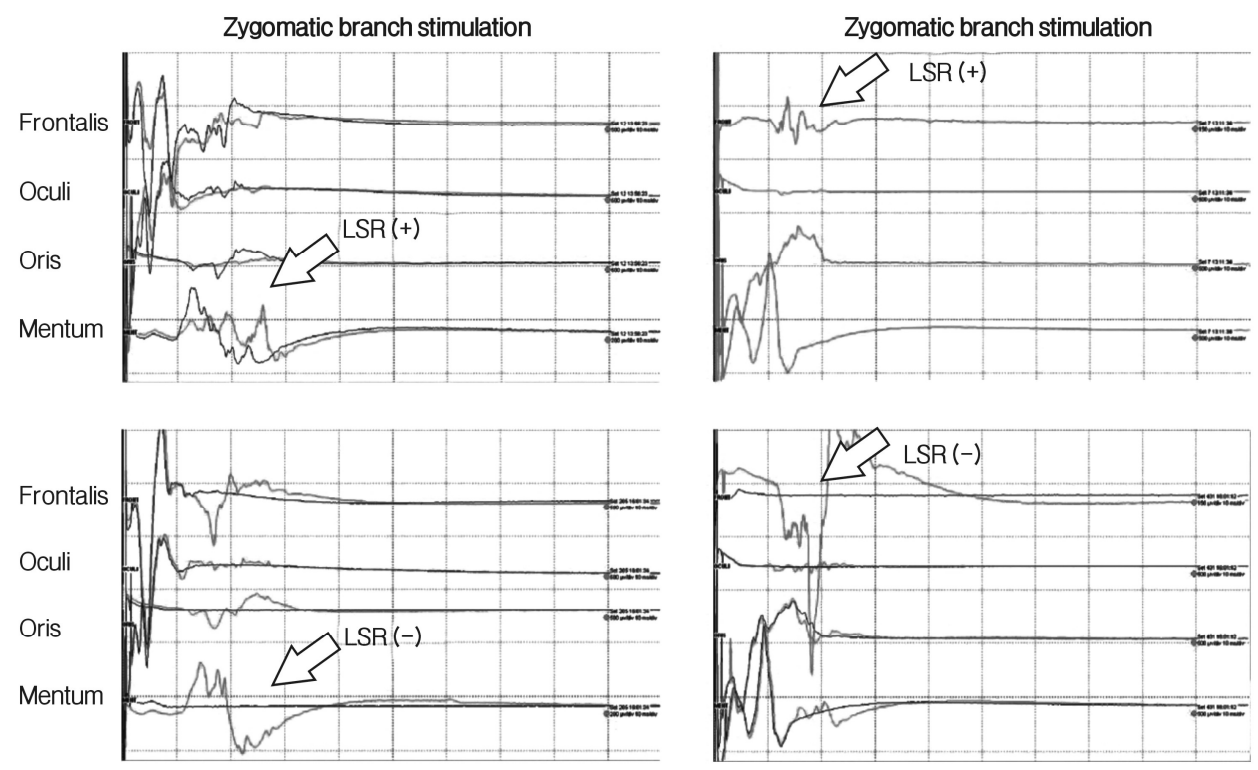

Fig. 2. Intra-operative neuro-monitoring showing lateral spread response (LSR) change. After performing microvascular decompression of the expected offending vessel, LSR persisted. As a result of further exploration, a small offender vessel was found, consistent with the fused image. The LSR disappeared after decompressing the small vessel.

(46.7\%) showed a concordant positive prediction result between both images (Table 1). Interestingly, the fused images revealed a secondary possible candidate offender other than main (predicted) offending vessel. Five cases were predicted to have another possible offender using the fusion technique (Table 2). Furthermore, the offenders in 4 cases were only predicted with fused MR images, and were not detected in conventional images (Table 1).

\section{Illustrative Case}

A 42-year-old man was admitted for right HFS. PICA was the only noticeable vessel contacting the facial nerve in the pre-operative conventional MR image. However, after performing MVD of the expected artery, lateral spread response (LSR) persisted in intra-operative neuromonitoring. As a result of further exploration, a small hidden offender vessel was found using a mirror view. The LSR disappeared after decompressing the small vessel (Fig. 2). Image fusion was performed post-operatively, and the small offending vessel seen during the intra-operative microscopic view was visualized in the fused image. Representative images and intra-operative views for neurovascular compression are shown (Fig. 3).

\section{DISCUSSION}

Diagnostic imaging modalities for many neurosurgical con-
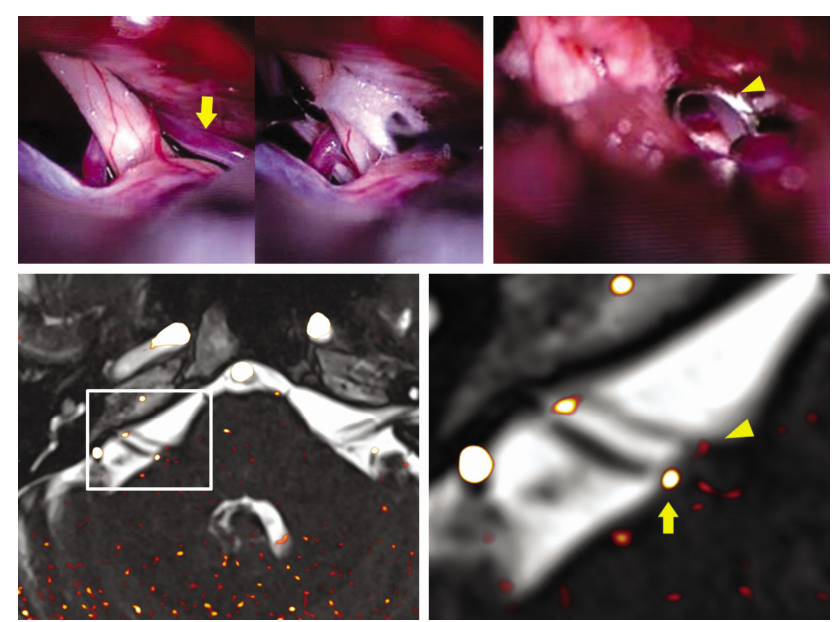

Fig. 3. Intra-operative microscopic views and fused images for the illustrative case. The right facial nerve was compressed by the right posterior inferior cerebellar artery (PICA) (arrow) at the root entry zone. Another small offender vessel (arrowhead) was found using the mirror view, which was consistent with the fused image.

ditions have been rapidly developing for decades. Several imaging methods have been used to identify areas of vascular compression, and MR tomography has emerged as the diagnostic tool of choice for this syndrome. Although MR imaging may be adequate for visualizing the cranial nerve and proximal vessels, it provides a somewhat crude estimate of the exact point of contact.

Lately, there have been a growing number of reports poin- 
ting out the accuracy of high-resolution $\mathrm{MR}$ imaging in predicting compression on the trigeminal or facial nerve root ${ }^{3,410,12)}$. Optimal imaging includes 3D CISS MR imaging and 3D TOF MRA, or its equivalent ${ }^{1-3,7-9,11,12)}$. The 3D CISS and 3D TOF MR images are complementary. The 3D CISS images clearly delineate structures (i.e., nerves or vessels) in the CSF space. How- ever, it is difficult to distinguish nerves from vessels according to signal intensity. In contrast, 3D TOF images show only vessels as high-signal-intensity areas in the CSF space, so the images are not adequate for displaying nerves. Therefore, combining these techniques complements the limitations of the other, and in many ways, capitalizes on the advantages of each. Especially, both the vessels and cranial nerves are visualized with superior clarity, and the location of contact between the vessel wall and the nerve can be more accurately pinpointed.

To fuse 3D TOF and 3D CISS images, OsiriX MD imaging software was used. OsiriX MD imaging software is capable of providing navigation and visualization of multimodal and multidimensional images. The interface is intuitive and interactive, and the reconstruction and fusion tools are fast and accurate. This system has proven very useful for image manipulation because it does not require a dedicated workstation. The images can be immediately processed and fused by the surgeon in a clinical environment or even in the operation room. The reason for modifying 3D TOF images by the software is that the original 3D TOF image does not have enough signal intensity to reveal small embedded vessels. However, there are possible drawbacks for this processing. In 3D TOF images, slow flow or flow from a vessel parallel to the scan-plane, may become desaturated just like stationary tissue, resulting in signal loss from the vessel. Morevover, turbulent flow may result in signal loss from the vessel and it can make false negative results. Lastly, there can be artifacts such as ghosting or susceptibility artifacts making false positive results.

Because MVD is a functional operation, it is ideal for the neurosurgeon to perform the procedure based on objective findings. However, sometimes the diagnosis of neurovascular compression is made light of the patient's symptoms and clinical course. Decompressive procedures have thus been performed without radiological evidence. While the conventional technique may provide useful information, significant neuroradiological experience is required to interpret the images and to identify the neurovascular structures involved. An imagefusion technique is more likely to depict a causative vessel in patients suffering from TN or HFS compared to conventional $\mathrm{MR}$ imaging in these cases, and it can be helpful to less-experienced, young neurosurgeons.

Some previous authors with experience operating on many patients have reported that in some patients, no causative ves- sels could be shown intra-operatively ${ }^{5,6}$. When a vascular structure is embedded in another solid structure, such as the brainstem, it is hardly visible in conventional MR images. This explains the findings for patients in whom no vascular compression was able to be defined. In our study, the illustrative case shows a similar situation. The small REZ artery embedded in the brainstem was not identifiable in the conventional image, but was identifiable after image fusion. Therefore, the use of the image-fusion technique might help in cases of conventional MR images with hidden vascular contacts.

To overcome prejudice, we matched each image using two different blind observers. The images were separately compared and cross-checked in a two-weeks interval. However, there still may exist observer bias in the interpretation. There were 2 cases that image-fusion techniques could not detect whereas the conventional imaging could detect. In one case, the offender was a vein, and the artery (PICA) in the other. The explanation for the undetected offenders is that sometimes in conventional MR imaging interpretation, we estimate the offender vessel by tracking the vessel route, not by exact contact in the REZ. However, by processing image-fusion technique, numerous small vessels in the REZ are exaggerated and it sometimes makes the observer difficult to define the offender vessel. The 3D TOF MRA has a weakness in that it cannot be used to visualize veins. We expect that further image-fusion studies using enhanced MR imaging will overcome this limitation. Our study was performed using a small number of patients, and has the structural limitation of being a retrospective study. A well-designed, prospective study is needed in the future.

\section{CONCLUSION}

An image-fusion technique can provide more visible information of possible offender vessels, and may play a valuable role in identifying critically embedded neurovascular structures for MVD surgery. This information can reassure surgeons and help them detect and localize additional offending vessels, even before a craniotomy. Therefore, the use of a pre-operative image-fusion technique might help in cases with hidden vascular contacts on conventional MR images.

\section{CONFLICT OF INTEREST}

None of the authors has any financial interest in the subject under discussion in this paper.

\section{DISCLOSURES}

No relevant nonfinancial relationship exists. 


\section{ACKNOWLEDGEMENTS}

We thank Wade Martin of Emareye for his critical review of this manuscript.

\section{REFERENCES}

1. Anderson VC, Berryhill PC, Sandquist MA, Ciaverella DP, Nesbit GM, Burchiel KJ: High-resolution three-dimensional magnetic resonance angiography and three-dimensional spoiled gradient-recalled imaging in the evaluation of neurovascular compression in patients with trigeminal neuralgia: a double-blind pilot study. Neurosurgery 58:666-673, 2006

2. Benes L, Shiratori K, Gurschi M, Sure U, Tirakotai W, Krischek $\mathrm{B}$, et al.: Is preoperative high-resolution magnetic resonance imaging accurate in predicting neurovascular compression in patients with trigeminal neuralgia? A single-blind study. Neurosurg Rev 28:131-136, 2005

3. Hastreiter P, Naraghi R, Tomandl B, Bonk A, Fahlbusch R: Analysis and 3-dimensional visualization of neurovascular compression syndromes. Acad Radiol 10:1369-1379, 2003

4. Kakizawa Y, Hongo K, Takasawa H, Miyairi Y, Sato A, Tanaka $\mathrm{Y}$, et al.: "Real" three-dimensional constructive interference in steady-state imaging to discern microneurosurgical anatomy. Technical note. J Neurosurg 98:625-630, 2003

5. Kaye AH: Trigeminal neuralgia: vascular compression theory. Clin Neurosurg 46:499-506, 2000
6. Klun B: Microvascular decompression and partial sensory rhizotomy in the treatment of trigeminal neuralgia: personal experience with 220 patients. Neurosurgery 30:49-52, 1992

7. Miller J, Acar F, Hamilton B, Burchiel K: Preoperative visualization of neurovascular anatomy in trigeminal neuralgia. J Neurosurg 108:477-482, 2008

8. Miller JP, Acar F, Hamilton BE, Burchiel KJ: Radiographic evaluation of trigeminal neurovascular compression in patients with and without trigeminal neuralgia. J Neurosurg 110:627632, 2009

9. Naraghi R, Hastreiter P, Tomandl B, Bonk A, Huk W, Fahlbusch $\mathrm{R}$ : Three-dimensional visualization of neurovascular relationships in the posterior fossa: technique and clinical application. J Neurosurg 100:1025-1035, 2004

10. Patel NK, Aquilina K, Clarke Y, Renowden SA, Coakham HB: How accurate is magnetic resonance angiography in predicting neurovascular compression in patients with trigeminal neuralgia? A prospective, single-blinded comparative study. Br J Neurosurg 17:60-64, 2003

11. Peker S, Dinçer A, Necmettin Pamir M: Vascular compression of the trigeminal nerve is a frequent finding in asymptomatic individuals: 3-T MR imaging of 200 trigeminal nerves using 3D CISS sequences. Acta Neurochir (Wien) 151:1081-1088, 2009

12. Yoshino N, Akimoto H, Yamada I, Nagaoka T, Tetsumura A, Kurabayashi T, et al.: Trigeminal neuralgia: evaluation of neuralgic manifestation and site of neurovascular compression with 3D CISS MR imaging and MR angiography. Radiology 228: 539-545, 2003 